\title{
ON THE EXISTENCE OF INVARIANT MEASURES
}

\author{
BY FRED B. WRIGHT ${ }^{1}$ \\ Communicated by P. R. Halmos, July 19, 1960
}

Let $T$ be a measurable transformation on a measure space $(\Omega, \propto, P)$, with $0<P(\Omega)<\infty$. Call $T$ absolutely continuous if $P(A)=0$ implies $P\left(T^{-1} A\right)=0$. The transformation $T$ is said to have the Birkhoff recurrence property if, for each $A \in Q, \lim _{n \rightarrow \infty}(1 / n) \sum_{j=0}^{n-1} \chi_{A}\left(T^{i} \omega\right)$ exists for almost all $\omega \in \Omega$. It has been shown that if $T$ is absolutely continuous and has the Birkhoff recurrence property, then there exists a non-negative, finite, countably additive measure $Q$ on $Q$ such that (i) $Q \ll P$, (ii) $Q$ and $P$ agree on invariant sets, (iii) $Q(A)=Q\left(T^{-1} A\right)$ for each $A \in Q$ [3]. In this paper we prove the following result.

THEOREM. If $T$ is an absolutely continuous measurable transformation on $(\Omega, a, P)$, where $0<P(\Omega)<\infty$, then there exists a non-negative, finite, finitely additive measure $Q$ with the following properties: (i) $P(A)$ $=0$ implies $Q(A)=0$; (ii) $Q$ and $P$ agree on invariant sets; (iii) $Q(A)$ $=Q\left(T^{-1} A\right)$ for each $A \in Q$.

We shall only outline the proof here. Let $B$ be the collection of all invariant sets; that is, $B \in \Theta$ if and only if $B=T^{-1} B$. Then $B$ is a $\sigma$-subalgebra of $a$. Consider the real algebras $L^{\infty}(Q)$ and $L^{\infty}(B)$, and represent them as the algebras $R(X)$ and $R(Y)$, respectively, of all continuous real-valued functions on the extremally disconnected, compact, Hausdorff spaces $X$ and $Y$. The Boolean algebras $E(X)$ and $E(Y)$ of idempotents in $R(X)$ and $R(Y)$ are both complete. Moreover there is a natural isomorphism of $R(Y)$ into $R(X)$ which maps $E(Y)$ into $E(X)$. The dual of this is a continuous mapping $\pi$ of $X$ onto $Y$, and the completeness of $E(Y)$ assures that the mapping is an open mapping. Theorems of Gleason [1] and Halmos [2] assert that $\pi$ has many cross-sections.

For any $f \in R(X)$ define functions $M f$ and $m f$ on $Y$ by setting $M f(y)=\operatorname{lub}\{f(x): \pi x=y\}$ and $m f(y)=\operatorname{glb}\{f(x): \pi x=y\}$. Since $\pi$ is open, both $M f$ and $m f$ are in $R(Y)$. Call a linear transformation $\mu: R(X) \rightarrow R(Y)$ a generalized mean if $m f \leqq \mu f \leqq M f$ for each $f \in R(X)$. Every cross-section of $\pi$ gives a mean that is a homomorphism. An absolutely continuous $T$ induces a linear transformation $t$ of $R(X)$ into itself. A generalized mean $\mu$ will be called invariant if $\mu t f=\mu f$ for each $f \in R(X)$. The set of all means is a nonempty, compact,

${ }^{1}$ Research Fellow of the Alfred P. Sloan Foundation. 
convex set in the weak operator topology on linear transformations from $R(X)$ to $R(Y)$. If $\mu$ is a mean, then $\mu t$ is also a mean, and the mapping $\mu \rightarrow \mu t$ is continuous. An application of the Tychonoff fixed point theorem yields a mean $\mu_{0}$ such that $\mu_{0} t=\mu_{0}$. That is, there is an invariant generalized mean.

Finally, any invariant generalized mean $\mu$ can be translated into a linear transformation of $L^{\infty}(Q)$ into $L^{\infty}(\beta)$, which will also be denoted by $\mu$. For each $A \in Q$, define $Q(A)=\int_{\Omega} \mu \chi_{A}(\omega) d P(\omega)$. Then $Q$ has all the required properties.

The transformation $T$ is said to have the Poincaré recurrence property if, for each $A \in Q, \sum_{j=1}^{\infty} \chi_{A}\left(T^{j} \omega\right)$ diverges for almost all $\omega \in A$. If an absolutely continuous $T$ has both the Poincaré and Birkhoff recurrence properties, then the countably additive and invariant $Q$ discussed above has the further property that $Q \equiv P$ [3]. It seems plausible that an absolutely continuous $T$ has the Poincare recurrence property if and only if any measure $Q$ satisfying the conditions of the theorem above has the further property that $Q(A)=0$ implies $P(A)=0$. This condition on $Q$ certainly implies the Poincare property; we have been unable to prove the converse.

The notion of generalized mean used in the proof above has a wide range of applicability, and can be placed in a more general setting. An account of this concept will be given elsewhere, along with a detailed proof of the theorem stated here. For the moment, we note only that, as it is used here, it is an extension of the concept of conditional expectation. It is the linearized form of the notion of a constant for a quantifier in algebraic logic [2], and is therefore the probabilistic analogue of this notion.

\section{REFERENCES}

1. A. M. Gleason, Projective topological spaces, Illinois J. Math. vol. 2 (1959) pp. 482-489.

2. P. R. Halmos, The representation of monadic Boolean algebras, Duke Math. J. vol. 26 (1959) pp. 447-454.

3. F. B. Wright, The converse of the individual ergodic theorem, Proc. Amer. Math. Soc. vol. 11 (1960) pp. 415-420.

TULANE UNIVERSITY 